\title{
Demographics of a large cohort of urban chronic hepatitis $\mathbf{C}$ patients
}

\author{
Firdous A. Siddiqui · Murray N. Ehrinpreis · James Janisse $\cdot$ Ravi Dhar · \\ Elizabeth May $\cdot$ Milton G. Mutchnick
}

Received: 15 February 2008/ Accepted: 19 June 2008/Published online: 25 July 2008

(C) Asian Pacific Association for the Study of the Liver 2008

\begin{abstract}
Purpose Recent studies suggest that African Americans (AA) with chronic hepatitis $\mathrm{C}$ (CHC) differ from nonHispanic whites (NHW) with respect to the natural history and mortality resulting from the complications of chronic liver disease. The aim of this study was to examine the demographics of a large cohort of CHC patients and identify potential differences between AA and NHW.

Methods This is a retrospective analysis, consisting of 2,739 hepatitis $\mathrm{C}$ antibody-positive patients seen at Wayne State University between 1995 and 2005. Patient demographics, risk factors, comorbidities, alanine aminotransferase (ALT), aspartate aminotransferase (AST), serum hepatitis C (HCV) RNA levels, genotype, and liver biopsy results were recorded. Results AA constituted 75.4\%, NHW 22.5\%, and Asians or Hispanics $2.1 \%$ of the patients. Males predominated (58\%), and the mean age of AA and NHW was 50.0 and 45.3 years, respectively $(P \leq 0.001)$. The most common risk factor was injection drug use in $55.3 \%$ (AA $57.1 \%$ vs. NHW 49.7\%; $P \leq 0.002$ ). HCV RNA by PCR obtained in 2,407 patients was positive in $94.8 \%$, with a high viral load in $61 \%$. Genotype 1 was significantly more frequent in AA $(92.6 \%)$ than in NHW $(70.6 \%, P \leq 0.001)$. AA had lower median ALT levels $(P \leq 0.001)$. In those patients who
\end{abstract}

F. A. Siddiqui $(\varangle) \cdot$ M. N. Ehrinpreis - R. Dhar · E. May ·

M. G. Mutchnick

Division of Gastroenterology, Wayne State University School of Medicine, Detroit, MI, USA

e-mail: Firdous.siddiqui@va.gov

J. Janisse

Division of Family Medicine and Public Health Sciences, Wayne

State University School of Medicine, Detroit, MI, USA were biopsied, there was no significant difference in fibrosis between the two groups. Aspartate to platelet index calculated in those patients who were not biopsied showed significantly lower fibrosis scores in AA.

Conclusions In this large cohort of $\mathrm{CHC}$ patients from a single institution, AA were older at presentation, had a higher prevalence of genotype 1, but significantly lower ALT levels than NHW.

Keywords Chronic hepatitis C · Demographics . African Americans

\section{Introduction}

Hepatitis $\mathrm{C}$ virus (HCV) infection is the most common chronic blood-borne infection in the United States, with an estimated 3.9-5 million people having been infected [1, 2]. $\mathrm{HCV}$ infection can be a progressive disease, resulting in cirrhosis that can lead to liver failure and/or hepatocellular carcinoma (HCC). Decompensated cirrhosis associated with $\mathrm{HCV}$ infection is the most frequent indication for liver transplantation in the United States and Europe [3].

There is significant racial disparity with respect to chronic hepatitis $\mathrm{C}$ (CHC) in the United States, with the highest prevalence of $\mathrm{HCV}$ infection in African Americans (AA) $(3.2 \%)$ and Hispanics $(2.8 \%)$ than non-Hispanic whites (NHW) (1.5\%) [2]. AA, representing $12-13 \%$ of the US population, account for $22 \%$ of the estimated 3.7 million Americans who have $\mathrm{CHC}$ viremia $[1,2]$. AA with $\mathrm{CHC}$ are reported to have lower pretreatment alanine aminotransferase (ALT) levels and less fibrosis than NHW [4]. Unfortunately, AA do not respond as well as NHW to interferon (IFN)-based therapies [5-10]. The explanation for this still remains unknown. 
Although epidemiological data on patients with $\mathrm{CHC}$ have been reviewed in the general population [2], and selected populations, such as veterans, prison inmates, injection drug users [5, 11], and Hispanics [12, 13], there are a few studies describing the demographics of AA patients with $\mathrm{CHC}$, and these have included only small numbers of patients [14-19].

Our objectives were to study the demographics of a large cohort of AA patients with $\mathrm{CHC}$ from a single urban medical center and to assess the clinical, biochemical, and histological differences between AA and NHW patients with CHC. Confirmation and understanding of these differences could provide insight into the differences in the natural history of the disease and, perhaps, the disparate response to IFN-based therapies between these 2 racial groups.

\section{Materials and methods}

\section{Patients}

The study population consisted of consecutive patients with a positive $\mathrm{HCV}$ antibody seen in our outpatient practice between January 1995 and July 2005. The majority of our patients $(78 \%)$ were referred by primary care physicians from within the city of Detroit and by the General Internal Medicine clinics from within our own institution. The remaining patients were referred by primary care physicians from other metropolitan Detroit communities. Referring physicians had uniform criteria for patient referral. A positive test for $\mathrm{HCV}$ antibody was the most common reason for referral. Data on all patients with $\mathrm{CHC}$ were entered into a computer database using unique identifier numbers for each patient and medical record numbers, thereby preventing duplication of patient data. Information on age, gender, race, and risk factors for $\mathrm{CHC}$, including injection drug use (IDU), intranasal drugs, blood transfusions, tattoos, multiple sexual partners, incarceration, hemodialysis, and other associated medical conditions, was obtained by experienced clinicians evaluating the patients. History of current and past alcohol intake was also recorded. These data were recorded on forms specifically created for this purpose and then entered into a computer database. Race was determined by self-reporting as AA, Asian, Hispanic, or NHW. The computer database was continually updated as data became available during patients' follow-up visits. The study was approved by the institutional review board at Wayne State University School of Medicine.

Methods

Anti-HCV serology was performed using the second generation ELISA for antibody (EIA II, Abbott Lab, Chicago,
IL). Testing for HCV RNA by reverse transcriptase-polymerase chain reaction (RT-PCR) was accomplished using the superquant assay (PCR range: $<200$ to $>2,000,000$ copies/ml; National Genetics Institute, CA) or the Amplicor HCV monitor test version 2.0 (PCR range: $<2,500$ to $>2,800,000 \mathrm{IU} / \mathrm{ml}$; Roche Molecular, Pleasonton, CA) performed in our laboratory. HCV genotype was tested using the line probe Versant Inno-LiPa HCV-II genotype assay (LiPA; Bayer Healthcare, Tarrytown, NY). Serum samples were also tested for antibodies to hepatitis A virus (anti-HAV), hepatitis B core antigen (anti-HBc), and hepatitis B surface antigen (HBsAg), alanine aminotransferase (ALT), aspartate aminotransferase (AST), and albumin. The Metavir score [20] for necroinflammation (grade $0=$ no inflammation, grade $1=$ mild inflammation, grade $2=$ moderate inflammation, and grade $3=$ severe inflammation) and fibrosis (stage $0=$ no fibrosis, stage $1=$ portal fibrosis, stage $2=$ periportal fibrosis, stage $3=$ bridging fibrosis, and stage $4=$ established cirrhosis) were recorded for all patients who underwent liver biopsy. In order to assess the fibrosis in the remaining patients, who did not agree to liver biopsy, an AST to platelet index (APRI) was calculated as follows: $\{(\mathrm{AST} / \mathrm{ULN}) / \mathrm{Plate}-$ lets $\left.\left(10^{9} / 1\right)\right\} 100$.

\section{Statistical analysis}

For comparison of categorical data, Pearson's chi-square or Fisher's exact tests were used. When AA and NHW patients differed across a set of categories, follow-up comparisons were made for each category separately. For normally distributed continuous data, $t$ tests were performed. For continuous data that did not meet the normality assumption, Mann-Whitney $U$ tests were performed. A $P$ value of $<0.05$ was considered statistically significant.

\section{Results}

Age, gender, and race

A total of 2,739 anti-HCV-positive patients were included in this analysis. As shown in Table 1, 2,035 patients were AA and 616 NHW. The remaining $2.1 \%$ were Asians and Hispanics. The selection criteria applied were identical in all groups. A predominance of male gender was noted. Nearly half of our patients were in their fifth decade at presentation, followed by those in their sixth decade. The mean age of AA patients was higher than that of NHW [49.98 and 45.34 years, respectively; $P \leq 0.001$ ]. The age distribution by decade is shown in Table 1 . Most patients reported high-risk behavior in their 20 s and taking into 
Table 1 Characteristics of chronic hepatitis C patients seen at Wayne State University, Detroit, MI, between 1995 and 2005

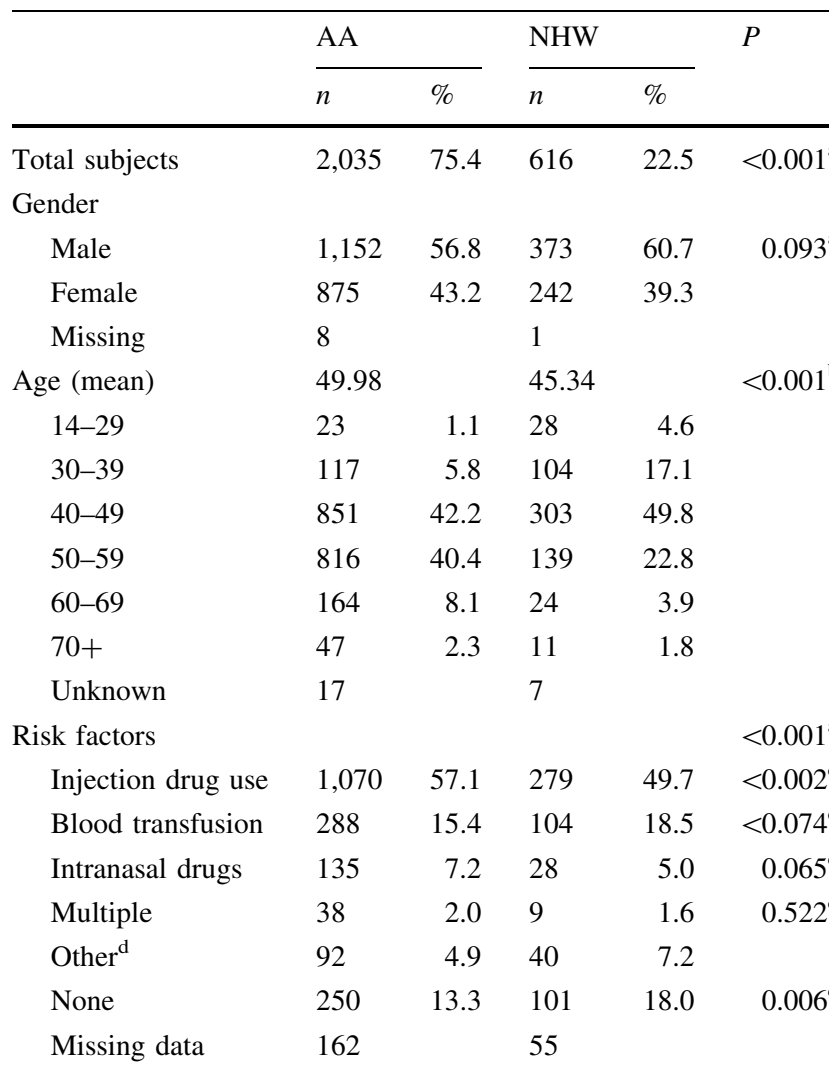

${ }^{a}$ Chi-square test for overall difference in categories for AA and whites

b $t$-Test for overall group in means for AA and NHW

c Chi-square test for differences in individual categories for AA and NHW

d Other includes employment in healthcare, hemodialysis, tattoos, multiple sexual partners, and a spouse with history of injection drug use

Abbreviations: AA, African Americans; NHW, non-Hispanic whites

account the average age of AA and NHW patients (49.98 and 45.34 years, respectively), we estimated the duration of disease to be approximately 20-25 years in most patients, which was generally similar in both groups.

\section{Risk factors}

IDU was identified in most patients (two-thirds of whom had multiple risk factors) and was the most common risk factor observed, followed by blood transfusion prior to 1992 and intranasal drug use (Table 1). A significantly higher proportion of AA were injection drug users $(P \leq 0.002)$. Fourteen percent of the patients had no identifiable risk factor. In the remaining patients, risk factors included employment in healthcare, hemodialysis, tattoos, multiple sexual partners, and a spouse with history of IDU. Alcohol consumption, both past and current, was also noted to be similar in both groups (data not presented).

Laboratory data and liver histology

As shown in Table 2, HCV RNA determination by PCR (qualitative or quantitative) was available for 2,407 patients, of whom 2,282 (95\%) were positive. There was no difference between AA and NHW in this regard. Quantitative PCR for HCV RNA was available for 1,928 patients. A high viral load was defined as more than 2,000,000 copies $/ \mathrm{ml}$ or $400,000 \mathrm{IU} / \mathrm{ml}$. Sixty-one percent of the patients had a high viral load. There was no significant difference in the viral load between AA and NHW. Genotype was available for 1,683 patients and the vast majority of AA were genotype $1(P \leq 0.001)$. Genotypes 2,3 , and 4 were also identified but much less frequently in AA than in NHW (Table 2).

The median ALT at presentation was significantly lower in AA (66 units/l, interquartile range $=44-96$ ) than in NHW (77, interquartile range $=50-121 \quad P \leq 0.001$ ). Mean serum albumin was significantly higher in NHW $(4.07 \mathrm{~g} / \mathrm{dl}, \quad \mathrm{SD}=0.36) \quad$ than $\quad \mathrm{AA} \quad(3.90, \quad \mathrm{SD}=0.7$; $P \leq 0.001$, data not shown). Although liver biopsy was offered to all patients who were seen, only 1,414 (51.6\%) patients $\quad(\mathrm{AA}=49.6 \%, \quad \mathrm{NHW}=57.8 \%) \quad$ underwent biopsy. Histological scoring showed lower grades of inflammation in AA than in NHW $(P=0.047)$. NHW had a higher median score for fibrosis than AA, although this did not achieve statistical significance $(P=0.17)$. The APRI evaluated in those patients who were not biopsied showed a significant difference $(P \leq 0.04)$, with a greater degree of fibrosis in NHW than in AA (Table 2).

Serological markers for hepatitis A and B

Serological markers indicating previous exposure to hepatitis $\mathrm{A}$ and $\mathrm{B}$ viruses were available in 1,740 and 1,841 patients, respectively, and showed the prevalence of both anti-HAV and anti-HBc antibodies to be significantly higher in AA than in NHW (Table 2). No difference in HBsAg prevalence was observed between the 2 groups.

\section{Discussion}

The explanation for the differences in the natural history and histological spectrum of $\mathrm{CHC}$ between AA and NHW is still not well established. One might have anticipated, on the basis of the decreased enzyme activity and less fibrosis that AA patients would have a more benign disease course. However, for reasons that are unclear, the complications of liver cirrhosis and the incidence of HCC are significantly 
Table 2 Laboratory data and liver histology of chronic hepatitis C patients seen at Wayne State University Hospital, Detroit, MI, between 1995 and 2005
${ }^{a}$ Chi-square test for overall difference in categories for AA and NHW

b High: $>2$ million copies $/ \mathrm{ml}$ or $>400,000 \mathrm{IU} / \mathrm{ml}$

c Fisher's exact test for overall difference in categories for AA and NHW

${ }^{\mathrm{d}}$ Chi-square test for differences in individual categories for AA and NHW

e Fisher's exact test for differences in individual categories for AA and NHW

${ }^{f}$ Mann-Whitney $U$ test for overall group in means for AA and NHW

g Includes those with clinically decompensated cirrhosis and those with $\mathrm{HCC}$

Abbreviations: AA, African Americans; NHW, nonHispanic whites

\begin{tabular}{|c|c|c|c|c|c|}
\hline & \multicolumn{2}{|l|}{ AA } & \multicolumn{2}{|c|}{ NHW } & \multirow[t]{2}{*}{$P$} \\
\hline & $n$ & $\%$ & $n$ & $\%$ & \\
\hline Positive HCV RNA by PCR (Qual + Quant) & & & & & $0.11^{\mathrm{a}}$ \\
\hline Positive & 1,703 & 95.1 & 507 & 93.4 & \\
\hline Negative & 87 & 4.9 & 36 & 6.6 & \\
\hline Viral load & & & & & $0.25^{\mathrm{a}}$ \\
\hline Low & 534 & 36 & 200 & 43 & \\
\hline High $^{\mathrm{b}}$ & 935 & 64 & 259 & 57 & \\
\hline Genotype & & & & & $<0.001^{\mathrm{c}}$ \\
\hline Type 1 & 1,171 & 92.6 & 262 & 70.6 & $<0.001^{\mathrm{d}}$ \\
\hline Type 2 & 66 & 5.2 & 40 & 10.8 & $<0.001^{\mathrm{d}}$ \\
\hline Type 3 & 18 & 1.7 & 61 & 16.4 & $<0.001^{\mathrm{d}}$ \\
\hline Type 4 & 9 & 0.7 & 8 & 2.2 & $<0.03^{\mathrm{e}}$ \\
\hline \multicolumn{6}{|l|}{ Missing data } \\
\hline \multicolumn{6}{|l|}{ Anti-HAV IgG (total) } \\
\hline Positive & 569 & 42.9 & 112 & 30.5 & $<0.001^{\mathrm{a}}$ \\
\hline Negative & 756 & 57.1 & 255 & 69.5 & \\
\hline Missing data & 710 & & 249 & & \\
\hline \multicolumn{6}{|l|}{ Anti-HBc IgG } \\
\hline Positive & 826 & 58.8 & 161 & 41.7 & $<0.001^{\mathrm{a}}$ \\
\hline Negative & 578 & 41.2 & 225 & 58.3 & \\
\hline Missing data & 631 & & 230 & & \\
\hline \multicolumn{6}{|l|}{ HBsAg } \\
\hline Positive & 57 & 3.6 & 21 & 4.9 & $0.24^{\mathrm{c}}$ \\
\hline Negative & 1,510 & 96.4 & 410 & 95.1 & \\
\hline Missing & 468 & & 185 & & \\
\hline \multicolumn{6}{|l|}{ Liver histology } \\
\hline Grade & & & & & $<0.04^{\mathrm{f}}$ \\
\hline 0 (No inflammation) & 77 & 8.4 & 23 & 7.3 & \\
\hline 1 & 454 & 49.2 & 138 & 44.1 & \\
\hline 2 & 312 & 33.8 & 117 & 37.4 & \\
\hline 3 & 79 & 8.6 & 31 & 9.9 & \\
\hline 4 & 0 & 0 & 4 & 1.3 & \\
\hline Stage & & & & & $0.16^{\mathrm{f}}$ \\
\hline 0 (No fibrosis) & 179 & 17.7 & 65 & 18.3 & \\
\hline 1 (Portal) & 274 & 27.2 & 83 & 23.3 & \\
\hline 2 (Periportal) & 180 & 17.8 & 62 & 17.4 & \\
\hline 3 (Bridging fibrosis) & 158 & 15.7 & 47 & 13.2 & \\
\hline 4 (Cirrhosis $^{\mathrm{g}}$ & 218 & 21.6 & 99 & 27.8 & \\
\hline APRI (mean) & 0.65 & & 0.78 & & 0.04 \\
\hline
\end{tabular}

higher in AA than in NHW (6.1 vs. 2.8/100,000 persons, respectively) [21-23]. AA men have a mortality rate from HCC that is twice the rate observed among NHW men [23, 24]. Previous studies evaluating these issues have been limited by the heterogeneity and small size of the populations studied [4, 14-17]. Our study of 2,739 patients with $\mathrm{CHC}$, of whom 2,035 were AA, constitutes the largest demographic analysis emerging from any single institution.
The higher prevalence of HCC in AA has been attributed by many investigators to a higher prevalence of coinfection with hepatitis $\mathrm{B}$ and hepatitis $\mathrm{C}$ because both entities are considered predisposing factors for the development of HCC. It is also conceivable that occult hepatitis $\mathrm{B}$ infection, that is, presence of anti-HBc antibody alone, could also contribute to the increased incidence of HCC in AA. In fact, we did observe a significantly higher 
prevalence in AA of serologic markers of previous exposure to hepatitis B and hepatitis A than NHW. In addition, lower socioeconomic status is correlated with both hepatitis A and B infections [25, 26]. Generally lower socioeconomic status in AA patients could explain the lower serum albumin levels observed, but we did not specifically evaluate socioeconomic status in our patient population.

Race and HCV genotype have important implications in the treatment of CHC. Genotype 1 has consistently been associated with a poorer response to IFN-based therapies, especially in AA [7-10, 19, 27-29]. A significantly higher prevalence of genotype 1 in AA than in NHW was observed in our cohort. Crosse et al. [15], Reddy et al. [18], and Fleckenstein [19] have also made similar observations. The question remains as to whether the increased prevalence of genotype 1 in AA patients reflects a host genetic susceptibility or reflects a difference in mode of transmission. We also noted a higher prevalence of genotype 3 in NHW (16.4\%) than $1.4 \%$ in AA. The reasons for these differences are unclear.

More recently Crosse et al. [15], in a cross-sectional analysis of $87 \mathrm{AA}$ and $136 \mathrm{NHW}$ with $\mathrm{CHC}$, showed lower fibrosis scores in AA. In a retrospective study of 355 patients $(\mathrm{AA}=112)$, Wiley et al. [4] evaluated the progression of histological changes in the liver of $\mathrm{CHC}$ patients over decades of exposure and noted that despite the longer duration of infection in AA, fibrosis scores were significantly lower than non-AA, especially in the third and fourth decades. Modes of transmission, estimated duration of infection, and alcohol abuse were similar in both groups. McHutchinson et al. [27] noted that AA were older at presentation, but these investigators did not observe a lower rate of cirrhosis. However, the number of AA in this large multicenter study was small, accounting for only $3 \%$ of the total patients enrolled. In contrast, Sterling et al. [16] in a study of 302 patients from a correctional facility $(\mathrm{AA}=49 \%)$ found the overall spectrum of HCV-related liver disease to be similar in AA and NHW, without significant differences in the total histological activity index (HAI) scores for fibrosis and inflammation. Although the utility of the APRI has been controversial [30], recent studies by Snyder et al. [31] and Shaheen and Myers [32] have described the APRI to be a good estimator of hepatic fibrosis and an important tool in excluding significant HCV-related fibrosis. Hence, in order to obtain an indirect measure of the liver fibrosis in those patients who were not biopsied, we utilized the APRI and noted significantly lower fibrosis scores in AA.

This is a retrospective study with inherent limitations. Although complete information was available regarding the race, gender, age, and risk factors, information about the viral load, genotype, and liver biopsy was not available for all patients. In addition, many patients seen between 1995 and 2000 did not have HCV RNA quantitation because access to this assay was not universally available. Even fewer patients had genotyping done. We believe the sampling obtained for each of these parameters was representative for both the NHW and the AA cohorts.

The importance of steatosis and insulin resistance on treatment response has gained emphasis more recently. Our data collection started in 1995 when these factors were not being evaluated, and hence this information was not recorded. Liver biopsy was recommended for all patients; however, those patients who did not wish to be treated often refused liver biopsy. Patient refusal of treatment and lack of adherence with follow-up visits were the 2 most common reasons for our inability to complete all components of the initial assessments.

In summary, the results derived from this large study of 2,739 patients with $\mathrm{CHC}$ confirm that AA when compared with NHW are older at presentation, are more likely to be injection drug users, infected with genotype 1, have lower ALT and albumin, and less hepatic fibrosis. AA also have a higher prevalence of previous exposure to hepatitis $A$ and B. Although these findings have been observed by other investigators in much smaller groups of AA, this report confirms these observations in a much larger group of AA seen in a single institution.

Ackowledgment We thank Dr. Jack Sobel from the Division of Infectious Diseases for his help and guidance in the preparation of the manuscript.

\section{References}

1. Edlin BR. Five million Americans infected with the Hepatitis C virus: a corrected estimate. Hepatology 2005;42(4, Suppl 1):44

2. Alter MJ, Jruzon-Moran MS, Nanina O, Mcquillan GM, Gao F, Margolis $\mathrm{H}$, et al. The prevalence of hepatitis $\mathrm{C}$ infection in the United States, 1988 through 1994. New Engl J Med 1999;341: 556-562

3. Poynard T, Yuen M, Ratziu V, Lai CL. Viral hepatitis C. Lancet 2003;362:2095-2100

4. Wiley TE, Brown J, Chan J. Hepatitis C infection in AfricanAmericans: its natural history and histological progression. Am J Gastroenterol 2002;97:700-706

5. Hepburn MJ, Hepburn LM, Cantu NS, Lapeer MG, Lawitz EJ. Differences in treatment outcome for hepatitis $\mathrm{C}$ among ethnic groups. Am J Med 2004;117:163-168

6. Samuel D. Chronic hepatitis $\mathrm{C}$ treatment patterns in AfricanAmericans: an update. Am J Gastroenterol 2005;100:716-722

7. Muir AJ, Bornstein JD, Killenberg PG. Atlantic coast hepatitis treatment group. Peginterferon alfa-2b and ribavirin for the treatment of chronic hepatitis $\mathrm{C}$ in blacks and non-Hispanic whites. N Engl J Med 2004;350(22):2265-2271

8. Kinzie JL, Naylor PH, Nathani MG, Peleman RR, Ehrinpreis MN, Lybik M, et al. African Americans with genotype 1 treated with interferon for chronic hepatitis $\mathrm{C}$ have a lower end of treatment response than Caucasians. J Viral Hepat 2001;8:264269 
9. Khan A, Mutchnick M, Ehrinpreis M, Dhar R, Siddiqui F. African-Americans with genotype-1 with chronic hepatitis $\mathrm{C}$ respond poorly to pegylated-interferon and ribavirin combination treatment as compared to Caucasians. Am J Gastroenterol 2005;100 (Suppl):311

10. Conjeevaram HS, Fried MW, Jeffers LJ, Terralt N, Wiley-Lucas TE, Afdal N, et al. Peg-interferon alfa-2a and ribavirin in African-American and Caucasian patients with chronic hepatitis C, genotype 1. Gastroenterology 2006;131(2):470-477

11. Nguyen HA, Miller AI, Dieperink E, Willenbring ML, Tetrick LL, Durfee JM, et al. Spectrum of disease in U.S. veteran patients with hepatitis C. Am J Gastroenterol 2002;97:1813-1820

12. Cheung RC, Currie S, Shen H, Ho SB, Bini EJ, Wright TL, et al. VA HCV-001 Study Group. Chronic hepatitis C in Latinos: natural history, treatment eligibility, acceptance, and outcomes. Am J Gastroenterol 2005;100:2186-2193

13. Celona AF, Yu MC, Prakash M, Kuo T, Bonacini M. Hepatitis C in a Los Angeles public hepatitis clinic: demographic and biochemical differences associated with race-ethnicity. Clin Gastroenterol Hepatol 2004;2:459-462

14. Howell C, Jeffers L, Hoofnagle J. Hepatitis C in African-Americans: summary of a workshop. Gastroenterology 2000;119:1385-1396

15. Crosse K, Onuora GU, Anania F, Laurin J, Papadimitriou J, Drachenberg C, et al. Racial differences in liver inflammation and fibrosis related to chronic hepatitis C. Clinical Gastroenterol Hepatol 2004;2:463-468

16. Sterling RK, Stravitz RT, Luketic VA, Sanyal AJ, Contos MJ, Mills AS, et al. A comparison of the spectrum of chronic hepatitis $\mathrm{C}$ virus between Caucasians and African Americans. Clin Gastroenterol Hepatol 2004;2:469-473

17. Pearlman BL. Hepatitis $\mathrm{C}$ virus infection in African Americans. Clin Infect Dis 2006;42(1):82-91

18. Reddy KR, Hoofnagle JH, Tong MJ, Lee WM, Pockros P, Heathcote EJ, et al. Racial differences in response to therapy with Interferon in CHC. Hepatology 1999;30:787-793

19. Fleckenstein J. Chronic hepatitis $C$ in African Americans and other minority groups. Curr Gastroenterol Rep 2004;6(1):66-70

20. Bedossa P, Poynard T, for the French METAVIR Cooperative Study Group. An algorithm for grading activity in chronic hepatitis C. Hepatology 1996;24:2889-2893
21. Man RE, Smart RG, Auglin L, Adlef EM. Reduction in cirrhosis deaths in the US: association with per capita consumption and AA membership. J Study Alcohol 1991;52:361-365

22. El Serag HB, Mason A. Rising incidence of HCC in US. N Engl J Med 1999;340:745-756

23. Nyugen MH, Whittemore AS, Garcia RT, Tawfeek SA, Ning J, Lam S, et al. Role of ethnicity in risk for hepatocellular carcinoma in patients with chronic hepatitis $\mathrm{C}$ and cirrhosis. Clin Gastroenterol Hepatol 2004;2:820-824

24. El-Serag HB. Hepatocellular carcinoma. Recent trends in United States. Gastroenterology 2004;127:S27-S34

25. Siddiqui F, Mutchnick M, Kinzie J, Peleman R, Naylor P, Ehrinpreis M. Prevalence of hepatitis A virus and hepatitis $B$ virus immunity in patients with polymerase chain reaction-confirmed hepatitis C: implications for vaccination strategy. Am J Gastroenterol 2001;96:858-863

26. Buffington J, Damon S, Moyer L, Culver D. CDC, Atlanta, GA. Racial differences in the knowledge regarding hepatitis $\mathrm{C}$ virus infection. JAMA 2000;284:1651-1652

27. McHutchinson JG, Poynard T, Pianko S, Gordon SC, Reid AE, Dienstag $\mathrm{J}$, et al. The impact of interferon plus ribavirin on response to treatment in black patients with chronic hepatitis $\mathrm{C}$. Gastroenterology 2000;119:1317-1323

28. Jeffers LJ, Cassidy W, Howell CD, Hu S, Reddy KR. Peginterferon alfa-2a $(40 \mathrm{kD})$ and ribavirin for black American patients with chronic HCV genotype 1. Hepatology 2004;39:1702-1708

29. Gish RG, Afdhal NH, Dieterich DT, Reddy KR. Management of hepatitis $\mathrm{C}$ virus in special populations: patient and treatment considerations. Clin Gastroenterol Hepatol 2005;3:311-318

30. Fontana RJ, Goodman ZD, Dienstag JL, Bonkovsky HL, Naishadham N, Sterling RK, et al. Relationship of serum fibrosis markers with liver fibrosis stage and collagen content in patients with advanced chronic hepatitis C. Hepatology 2008;47:789-798

31. Snyder N, Gajula L, Xiao SY, Grady J, Luxon, Lau DT, et al. APRI: an easy and validated predictor of hepatic fibrosis in chronic hepatitis C. J Clin Gastroenterol 2006;40(6):535-542

32. Shaheen AA, Myers RP. Diagnostic accuracy of the aspartate aminotransferase to platelet ratio index for the prediction of hepatitis C-related fibrosis: a systematic review. Hepatology 2007;46(3):912-921 